\title{
Further Observations on the Usefulness of the Sonographic Murphy Sign in the Evaluation of Suspected Acute Cholecystitis
}

\author{
Robert L. Bree, MD
}

\begin{abstract}
A positive sonographic Murphy sign, the presence of maximal tenderness elicited over a sonographically localized gallbladder, has been reported to be a helpful adjunctive finding in patients with proven acute cholecystitis who are evaluated with ultrasonography. We evaluated 200 patients with right upper quadrant pain, thought to be acute cholecystitis. Results of ultrasound examinations and subsequent follow-up were tabulated. The sensitivity of the sonographic Murphy sign in acute cholecystitis was $86 \%$ with a specificity of $35 \%$, positive predictive value of $43 \%$, and negative predictive value of $82 \%$. The sensitivity of the sonographic findings, including stones, gallbladder wall edema, and pericholecystic fluid collections, was $93 \%$, a specificity of $53 \%$. The combination of the Murphy sign accompanied by gallstones yielded a specificity of $77 \%$. The large number of false positives, and only moderate improvement in specificity when accompanied by gallstones, makes this sign unreliable in separating acute from chronic cholecystitis. (C) 1995 John Wiley \& Sons, Inc.

Indexing Words: Gallbladder · Cholecystitis · Murphy sign
\end{abstract}

There has been great debate in the literature about the diagnostic procedure of choice in acute cholecystitis. Sonography has been favored by some as the first choice in evaluating suspected acute cholecystitis. ${ }^{1-5}$ In the proper clinical setting, the detection of gallstones is useful in diagnosing acute cholecystitis, but can fail because many patients with clinically innocuous gallstones may have right upper quadrant pain which is unrelated to the presence of the stones. ${ }^{1-5}$

It has been proposed that the presence of a positive sonographic Murphy sign (SMS), defined as maximal tenderness elicited over a sonographically localized gallbladder, is a useful adjunct in the evaluation of the patient with suspected acute cholecystitis. ${ }^{2,6,7}$ It was hoped that this sign would help differentiate patients with acute cholecystitis from those with chronic cholecystitis. ${ }^{6}$ We have observed a large number of falsepositive SMS in our practice. We therefore under-

From the Department of Radiology, University of Michigan Medical Center, 1500 E. Medical Center Drive, Ann Arbor, Michigan. For reprints contact Robert L. Bree, MD, Department of Radiology, University of Michigan Medical Center, 1500 E. Medical Center Drive, Ann Arbor, MI 48109/0030. took a study evaluating the SMS in a large consecutive group of patients.

\section{MATERIALS AND METHODS}

We examined 200 consecutive patients referred for evaluation of clinically suspected acute cholecystitis. All patients were from the emergency room or recently admitted to the hospital and all were awake and alert. The patients underwent sonography with evaluation of the SMS. Various real-time scanners were used for obtaining the sonograms. Sonographic findings positive for the possible presence of acute cholecystitis included the presence of calculi with or without gallbladder wall thickening or pericholecystic fluid. Examinations were performed by registered sonographers. If the SMS was questionable, physician input was elicited. The SMS was considered positive if the patient identified the point of maximal tenderness as a point over the real-time sonographically localized gallbladder. The SMS was considered negative if the patient identified an area other than that over the gallbladder as the area of greatest tenderness, could not identify an area of 
greatest tenderness, or had no tenderness. The combination of gallstones and a positive SMS was analyzed statistically to see if there was any diagnostic enhancement. The SMS was evaluated separately from the presence or absence of a sonographically abnormal gallbladder.

One hundred three (103) patients underwent surgery and 97 were treated without surgery. Patients had pathologic confirmation of acute cholecystitis only when a surgical specimen was obtained. Acute cholecystitis was deemed present when it was confirmed pathologically by the identification of either hemorrhagic necrosis of the gallbladder wall, transmural inflammation, or mural edema. ${ }^{2}$ These histologic criteria are considered strict when compared to those used by others, which include neutrophilic infiltration of the mucosa or submucosa. The remaining patients had an alternate diagnosis confirmed by another modality as the cause for the right upper quadrant pain or their condition was followed until it resolved.

\section{RESULTS}

Pathological evidence of acute cholecystitis was obtained in 73 patients. One hundred twentyseven (127) patients had other etiologies for the acute abdominal pain. Thirty were proven by surgery and 97 by clinical observation or nonsurgical diagnostic procedures. The results are shown in Tables 1 and 2, separating surgical and nonsurgical cases. The sensitivity of the sonographic Murphy sign for acute cholecystitis was $86 \%$, with a specificity of $35 \%$ and overall accuracy of $54 \%$. The positive predictive value for the SMS was $43 \%$ and the negative predictive value was $82 \%$. The sensitivity of the ultrasound findings was $93 \%$, with a specificity of $53 \%$ and overall accuracy of $68 \%$. The sensitivity of the SMS for

TABLE 1

Results of Imaging Studies on Patients with Acute Right Upper Quadrant Pain

\begin{tabular}{lcc}
\hline & $\begin{array}{c}\text { Acute } \\
\text { Cholecystitis* }\end{array}$ & $\begin{array}{c}\text { Not Acute } \\
\text { Cholecystitis* }\end{array}$ \\
\hline $\begin{array}{l}\text { Sonographic Murphy } \\
\text { sign positive }\end{array}$ & $\%$ & $\%$ \\
Sonographic Murphy & 86.3 & 65.4 \\
$\quad$ sign negative & 13.7 & 34.6 \\
Ultrasound positive & 93.2 & 47.2 \\
Ultrasound negative & 6.8 & 52.8 \\
Gallstones + SMS & 69.9 & 22.8 \\
\hline
\end{tabular}

*Number: acute cholecystitis, 73; not acute cholecystitis, 127 . Total: 200 patients.
TABLE 2

Results of Imaging Studies on Patients with Acute Right Upper Quadrant Pain Followed by Surgery

\begin{tabular}{lcc}
\hline & $\begin{array}{c}\text { Acute } \\
\text { Cholecystitis }\end{array}$ & $\begin{array}{c}\text { Not Acute } \\
\text { Cholecystitis* }\end{array}$ \\
\hline $\begin{array}{l}\text { Sonographic Murphy } \\
\quad \text { sign positive }\end{array}$ & $\%$ & $\%$ \\
Sonographic Murphy & 86.3 & 63.3 \\
$\quad$ sign negative & 13.7 & 36.7 \\
Ultrasound positive & 93.2 & 83.3 \\
Ultrasound negative & 6.8 & 16.7 \\
Gallstones + SMS & 69.9 & 46.7 \\
\hline
\end{tabular}

*Number: acute cholecystitis, 73; not acute cholecystitis, 30. Total: 103 patients.

acute cholecystitis when accompanied by the presence of gallstones was $70 \%$. The specificity of this combination was $77 \%$, with a positive predictive value of $64 \%$ and negative predictive value of $82 \%$. The overall accuracy was $75 \%$. All 10 of the false-negative SMS had positive ultrasound examinations. Seven of the 10 with negative SMS had gangrenous cholecystitis histologically. Conversely, all 5 false-negative ultrasound examinations had positive SMS. Fourteen of the 30 patients undergoing surgery who did not have acute cholecystitis had gallstones and a positive sonographic Murphy sign. Tables 3 and 4 show the patients without acute cholecystitis with and without surgery and the results of the SMS for the most frequent diagnosis.

\section{DISCUSSION}

In our study, sonography had a high sensitivity (93\%) for the diagnosis of acute cholecystitis. Unfortunately, the specificity is relatively low (53\%), but consistent with previous results. ${ }^{2,5,6}$ Ultrasonography is unable to differentiate between calculi that are associated with acute cholecystitis and calculi that are present in a patient with an-

TABLE 3

Murphy Sign Results in Diagnoses Other Than Acute Cholecystitis

\begin{tabular}{lcc}
\hline & \multicolumn{2}{c}{ Murphy Sign } \\
\cline { 2 - 3 } Final Diagnosis* & Positive & Negative \\
\hline & $\%$ & $\%$ \\
Chronic cholecystitis (35) & 62.9 & 37.1 \\
Pain unknown etiology (23) & 73.9 & 26.1 \\
Gastroenteritis (16) & 43.8 & 56.2 \\
Pancreatitis (13) & 46.2 & 53.8 \\
Ulcer (8) & 37.5 & 62.7 \\
Other (32) & 78.1 & 21.9 \\
\hline
\end{tabular}

*Number given in parentheses. Total: 127 patients. 
TABLE 4

Murphy Sign Results in Surgical Diagnoses Other Than Acute Cholecystitis

\begin{tabular}{lcc}
\hline & \multicolumn{2}{c}{ Murphy Sign } \\
\cline { 2 - 3 } \multicolumn{1}{c}{ Final Diagnosis* } & Positive & Negative \\
\hline & $\%$ & $\%$ \\
Chronic cholecystitis (24) & 62.5 & 37.5 \\
Pain unknown etiology (3) & 100.0 & 0.0 \\
Pancreatitis (2) & 50.0 & 50.0 \\
Ulcer (1) & 0.0 & 100.0 \\
\hline
\end{tabular}

*Number given in parentheses. Total: 30 patients.

other etiology for the acute abdominal pain, including chronic cholecystitis. It was hoped that the SMS would help increase the specificity by differentiating pain caused by acute cholecystitis from pain caused by another etiology. ${ }^{6}$

Our results of the SMS are not as good as others have reported. Ralls et al. ${ }^{5}$ reported sensitivity of $72 \%$, specificity of $88 \%$, and accuracy of $79 \%$. They used more liberal criteria for the pathological diagnosis of acute cholecystitis. These include transmural inflammation of the gallbladder, hemorrhagic mural necrosis, or significant acute neutrophilic infiltration of the muscularis or mucosa. By using liberal criteria for the pathologic diagnosis of acute cholecystitis and a well-screened population, a $52 \%$ incidence of acute cholecystitis was obtained. Laing et al. ${ }^{2}$ had an incidence of acute cholecystitis of $36 \%$ (identical to our incidence of $36 \%$ ). They used a stricter definition of acute cholecystitis that was similar to ours. It is possible that some of the patients in our series treated without surgery might have had acute cholecystitis, but if they were treated in this fashion, it was usually because of a negative or equivocal cholescintigram and resolving symptoms. Therefore, neither ultrasound examination nor the SMS is capable of separating the surgical from the nonsurgical patient.

Laing et al. ${ }^{2}$ reported results of $76 \%$ sensitivity, $94 \%$ specificity, and accuracy of $86 \%$ for acute cholecystitis with the SMS. They used the SMS in conjunction with the presence of calculi and not as an isolated finding. These results were only slightly better than ours and not as good as others using ultrasonography without the SMS. ${ }^{3,4}$ Our larger patient population and the obvious subjectivity of the SMS can help explain the discrepancy, even though they used the stricter pathologic criteria for acute cholecystitis. Ralls et al. ${ }^{5}$ also combined stones with the SMS and obtained a positive predictive value of $90 \%$. When our data using a combination of SMS and gallstones are analyzed, we have a similar sensitivity, but our specificity remains rather low at $77 \%$ and our positive predictive value was $64 \%$. This continues to indicate relatively poor performance of the SMS. In a separate study, Ralls et al. ${ }^{6}$ reported a sensitivity of $63 \%$, specificity of $94 \%$, accuracy of $87 \%$, and positive predictive value of $73 \%$ for acute cholecystitis with the SMS. In their study, they excluded 167 of the original 427 patients because they were lost to follow-up. The wide range of results reported for the SMS indicate that this sign has a significant operator-specific variability and that it is difficult to reproduce the results.

Simeone et al. ${ }^{8}$ showed that only $33 \%$ of the patients with gangrenous cholecystitis had a positive sonographic Murphy sign. This is believed to be due to the loss of innervation caused by the inflammation destroying the nerves to the gallbladder. This may help explain the negative SMS in 10 (14\%) of our patients with acute cholecystitis. Of the 10 false negatives in our study, 7 had acute gangrenous cholecystitis.

The sonographic Murphy sign relies on the patient to identify an area of greatest tenderness. To be considered positive, the surgical Murphy sign relies only on the cessation of breathing on deep inspiration, with the examiner's hand palpating the subcostal right upper quadrant. The sonographic Murphy sign does not employ the deep breath technique described with the original Murphy sign. The sonographic Murphy sign was positive, as well as negative, in a wide variety of disease processes, including ulcer, angina pectoris, pancreatitis, gastroenteritis, ventral hernia, ectopic pregnancy, urinary tract infections, bowel obstruction, cancer of the gallbladder, hepatitis, esophageal dysmotility, herpes zoster, ovarian cyst, pelvic inflammatory disease, and rib fracture. In 5 patients with acute cholecystitis, the SMS was positive when the sonogram was negative. The SMS was helpful in this group, but all patients subsequently had a positive cholescintigram. The SMS can be positive with almost any abdominal pathology that can produce pain. This appears to be related to the fact that abdominal pain of any source can be difficult for a patient to localize. This is especially true if the patient has a process that may spread inflammation around the gallbladder or to other regions in the abdomen.

The sonogram has a high sensitivity $(93 \%)$ but a low specificity (53\%), leading to a low accuracy of $68 \%$. This was caused by the inability of ultrasonography to differentiate between acute cholecystitis and the incidental finding of gallstones in a patient with another source of right upper quad- 
rant pain. Only the infrequent presence of gallbladder wall edema and pericholecystic fluid with associated stone can allow a more accurate diagnosis of acute cholecystitis. Because of its sensitivity, availability, and noninvasiveness, ultrasonography remains an excellent first step in evaluating a patient who clinically appears to have acute cholecystitis.

We conclude that the sonographic Murphy sign does not add significant information over ultrasound examination alone in the diagnosis of acute cholecystitis. With a positive predictive value of $43 \%$, a large number of patients may be falsely assumed to have acute cholecystitis when more objective findings such as a positive cholescintigram may be necessary. Operator and patient variability makes the SMS difficult to accurately reproduce. The SMS does not compare technically to the original Murphy sign. Ultrasonography is still the best modality to screen for acute cholecystitis, but suffers from low specificity.

\section{REFERENCES}

1. Fink-Bennett D, Freitas J, Ripley S, et al: The sensitivity of hepatobiliary imaging and real-time ul- trasonography in the detection of acute cholecystitis. Arch Surg 120:904-906, 1985.

2. Laing F, Federle M, Jeffery B, et al: Ultrasonic evaluation of patients with acute right upper quadrant pain. Radiology 140:449-455, 1981.

3. Martinez A, Bona X, Velasco M, et al: Diagnostic accuracy of ultrasound in acute cholecystitis. Gastrointest Radiol 11:334-338, 1986.

4. Ralls P, Colletti P, Halls J, et al: Prospective evaluation of $99 \mathrm{mTc}$-IDA cholescintigraphy and grayscale ultrasound in the diagnosis of acute cholecystitis. Radiology 144:369-371, 1982.

5. Ralls P, Colletti P, Lapin S, et al: Real-time sonography in suspected acute cholecystitis: prospective evaluation of primary and secondary signs. Radiology 155:767-771, 1985.

6. Ralls P, Halls J, Lapin S, et al: Prospective evaluation of the sonographic Murphy sign in suspected acute cholecystitis. $J$ Clin Ultrasound 10:113-115, 1982.

7. Ulreich S, Foster K, Stier S, et al: Acute cholecystitis: comparison of ultrasound and intravenous cholangiography. Arch Surg 115:158-160, 1980.

8. Simeone J, Brink J, Mueller P, et al: The sonographic diagnosis of acute gangrenous cholecystitis: importance of the Murphy sign. AJR 152:289-290, 1989. 